\title{
LDWS For Smart Vehicles Using Image Processing Based On Hough Transform
}

\author{
Rajashri Gulve ${ }^{1}$, Anuja Diggikar ${ }^{2}$ \\ Research Scholar, Department of E\&TC, SPWE College, Aurangabad, India ${ }^{1}$ \\ Professor, Department of E\&TC, SPWE College, Aurangabad, India ${ }^{2}$
}

\begin{abstract}
This paper proposes a method for monitoring the position of a vehicle on a lane. Cameras are mounted on the sides of vehicle and focus downwards on the road to capture images containing the lane lines and wheels/vehicle boundaries. The Hough transform (HT) is employed to detect the lane from the captured images. The correlation of sequential images and the constraints of lane lines and cameras are used to minimize the region of interest (ROI) in both image space and HT space, which increases the capture accuracy and decrease the computation complexity. The lane position monitoring system is assumed to be useful for unmanned vehicles, and can also provide warnings in normal vehicles to enhance driving safety. The experiments show the method is effective on various roads and driving situations.
\end{abstract}

Keywords: Hough Transform; Lane line detection; Video content analysis; Unmanned vehicle

\section{INTRODUCTION}

Nowadays, the growing volume of the traffic all around the world requires higher levels of the traffic safety. On the road, there are so many unsafe driving cars that the driver requires more careful while driving. Important for driver is being careful when he/she must change lane, especially in new driver who absolutely cannot keep too much information at once and has no confidence for driving. Driver may be loss of concentration and control car.In fact, human behaviors are indeed hard to recognize, predict and handle by current available equipment's. Therefore, a monitoring and warning system focusing on the vehicle behaviors is needed while the car is moving on the road. A Hough Transform Based Lane Detection for Driving System was developed to find the way that can reduce the loss of views to the front street. The Hough Transform, one of the easy techniques for image processing, is used for detecting lane road within an image. The system is designed to work in conjunction with the general principles of the webcam image processing. The paper helps the driver by increasing decision-making while driving in order to monitor the movement of vehicles through the lanes.

In This Paper, the lane detection algorithm is proposed by using information of camera and road.

Firstly, the acquired video file is extracted to image frames. Secondly, the image frames are specifically divided into road part from the information of webcam camera. Thirdly, RGB color image is converted by image enhancement to grayscale image. Threshold is applied to evaluate the grayscale image into a binary image. Fourthly, the Hough transform method is developed to detect the lanes in the road image. The concept of this paper is to provide a computer to monitor the image of the road and detect its lanes in various areas for improving the safety driving In earlier system such as $\mathrm{Cl}^{\prime}$ audio and Christa proposed a lane departure warning system that estimates lane orientation through linear parabolic model.
B. Yu, W. Zhang and Y. Cai used a Gaussian template to remove the dirty spots in the image and dynamical threshold choosing to find lane marks. TLC and CCP method are used to make lane departure decision. P.Y. Hsiao, C. W. Yeh, S. S. Huang and L. C .Fu proposed an embedded ARM-based real-time LDWS. W. Zhu, F. Liu, Z. Li, X. Wang and S. Zhang proposed an algorithm to chooses a common curved lane parameter model which can describe both straight and curved lanes.

N. M. Enache used composite Lyapunov Functions, polydral-like invariant sets and linear matrix inequality(LMI) method to implement the lane-departure avoidance system. M.J Jeng proposed a LDWS which is implemented in hardware and software. The hardware is implemented by FPGA. H. Y. Cheng proposed a method to utilize size, shape, and motion information to find the lane mark out. A .AM. Assidiq proposed a method to detect the lane mark by Canny edge detector and Hough Transform. J. F. Liu used gradient to find out the both lane marks.

\section{PROPOSED METHODOLOGY}

The main components of driving system detection based on the Hough transform are described as below:

Step 1:

Receiving image: the developed system is able to acquire images from video files, which are already saved in a form of avi video. The image will be the same size as the resolution of the camera that used to record without any additional configuration.

\section{Step2:}

Splitting image: The system analyzes the video file to apply to the video frames splitting process by applying the data of video files and then the system specifies the variable to divide sub frames in the variable of array image to the frame. 


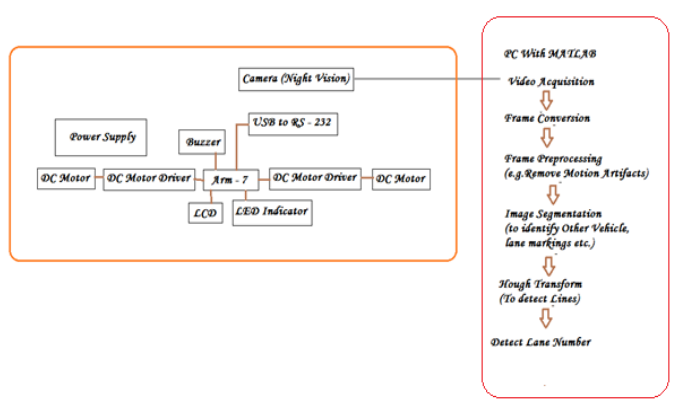

Fig. 1 Proposed system

Step3:

Cropping image: Specifying the area of interest by using crop tool to extract the region of road part within the image. It can specify the size and position as parameters by specifying the crop rectangle as a four-element position vector.

Step4:

Image enhancement (from RGB color to grayscale image): In this process, the system conducts the frame, which stored in the storage module, to convert RGB color image into grayscale image. After this process, we have the grayscale image.

Step5:

Converting grayscale image to binary image: Threshold is an appropriate method that uses to convert the grayscale image to binary.

Step6:

Segmenting and removing objects: Using the opening operation, Morphological Operations, which can assist segmentation and remove objects which are not structuring element.

Step7:

Defining the analytic area within the image: The system conducts the binary image to define detection or surveillance area within the image by using the Hough transform which can identify straight line. We set green color to detect line and red color to detect the longest line segment.

Step8:

Computing Hough transform: From step 7, the system operates the defined segment to analyse the Hough transform. The original image is determined by Hough function

After computing, we get the Hough transform in red color to show the line parameters of its algorithm in the image. This is the flowchart for proposed system of lane departure warning system for smart vehicles.

In this system, firstly camera captures images, then images gets enhanced and processed. Then system checked whether road lines are introduced or not. If yes then system continue next step. Or if no then it again returns to strat. then it shows lines.

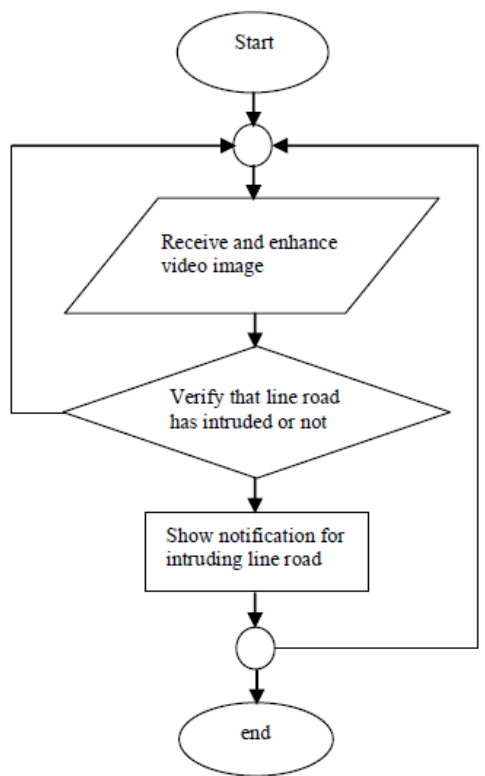

Fig.2 Proposed system algorithm

\section{CONCLUSION}

In this paper, we proposed a method for helping the drivers in the lane departure decision-making based On the Hough transform by detecting lanes. The adopted lane detection method was consisted of image Processing, morphological operations, dynamical threshold, Hough lines and Hough transform. Its advantages of Hough transform are easy to use, low cost and also effective in detect lines form the image. Experimental results reveal the efficiency of the performance of the lane detection algorithm in various environments. In further research, we will focus on how to detect the lane correctly in various situations.

\section{REFERENCES}

[1] R. Rajamani, T. Han-Shue, L. Boon Kait, Z. Wei-Bin, "Demonstration of integrated longitudinal and lateral control for the operation of automated vehicles in platoons", IEEE. Trans. Control Systems Technology, vol. 8, pp.695-708, July2000.

[2] M. Bertozzi and A. Broggi, "GOLD: A parallel real-time stereo vision system for generic obstacle and lane detection," IEEE Trans. On Image Processing, vol. 7, pp. 62-81, Jan. 1998

[3] M. Bertozzi and A. Broggi, and A. Fascioli, "Obstacle lane detection on ARGO autonomous vehicle," in Proc. IEEE Intelligent Transportation System Conf. '97, Boston, MA.pp.1010-1015.

[4] M. Bertozzi and A. Broggi, "Vision-Based Vehicle Guidance," Computer, vol. 30, pp.49-55, July. 1997

[5] R. Chapuis, J. Gallice, F. Jurie, and J. Alizon, "Real time road mark following”, Signal Processing, vol. 24, pp. 331- 343, Dec. 1991.

[6] http://www.IVsource.net

[7] http://www.citroen.com/CWW/en-US/TECHNOLOGIES /SECURITY/AFIL/

[8] M. Donath, "The Vehicle-Highway Partnership: The Infrastructure Needs to Get Smarter," ITS Institute, University of Minnesota, Proceedings of the 7th International Task Force on Vehicle-highway Automation Paris, 2003.

[9] B. Richard, "Intelligent Vehicle Technology and Trends", pp. 99 http://www.moi.gov.tw/stat/english/index.asp

[10] M. Fitzgerald, "Lane Departure Warning boots Anti-Rollover Protection", Strategy Analytics in Automotive Design Line, http://www.automotivedesignline.com/howto/60405845

[11] J. Goldbeck and B. Huertgen, "Lane detection and tracking by video sensors," in Proceedings of IEEE Intelligent Transportation Systems, vol. 1, October 1999, pp. 74-79. 
International Journal of Advanced Research in Computer and Communication Engineering Vol. 3, Issue 10, October 2014

[12] J. Park, J. Lee, and K. Jhang, "A lane-curve detection based on an LCF,” Pattern Recognition Letters, vol. 24, no. 14, pp. 2301-2313, October 2003

[13] Y. Wang, E. Teoh, and D. Shen, "Lane detection and tracking using B-snake," Image and Vision Computing, vol. 22, no. 4, pp. 269280, April 2004.

[14] R. Chapuis, R. Aufrere, and F. Chausse, "Accurate road following and reconstruction by computer vision," IEEE Trans. Intelligent Transport. Systems, vol. 3, pp. 261-270, Dec. 2002.

[15] A. Broggi, M. Bertozzi, Lo Guarino, C. Bianco, and A. Piazzi, "Visual perception of obstacle and vehicles platooning," IEEE Trans. Intelligent Transport. Syst., vol. 1, no. 3, pp. 164-176, Sept. 2000 